\title{
The protoDUNE-SP experiment and its prompt processing system
}

\author{
Maxim Potekhin ${ }^{* \dagger}$ \\ Brookhaven National Laboratory, USA \\ E-mail: potekhin@bnl.gov
}

The Deep Underground Neutrino Experiment (DUNE) will employ a uniquely large Liquid Argon Time Projection Chamber with four separate modules of 10kt fiducial mass each. Different modules will utilize single and dual-phase Liquid Argon technologies. An experimental program ("protoDUNE") has been initiated which includes a beam and cosmic ray test of large-scale DUNE prototypes at CERN in 2018. The volume of data to be collected by the protoDUNE single-phase detector will amount to a few petabytes and the sustained rate of data sent to mass storage will be in the range of a few hundred megabytes per second. The protoDUNE experiment requires substantial Data Quality Monitoring capabilities in order to ascertain the condition of the detector and its various subsystems. To this end, a Prompt Processing system has been developed which is complementary to Online Monitoring and is characterized by a lower bandwidth, scalable CPU resources and end-to-end latency on the scale of a few minutes. We present the design of the protoDUNE Prompt Processing system, the current status of its development and testing and issues related to its interfaces and deployment.

EPS-HEP 2017, European Physical Society conference on High Energy Physics

5-12 July 2017

Venice, Italy

${ }^{*}$ Speaker.

${ }^{\dagger}$ For the Deep Underground Neutrino Experiment Collaboration. 


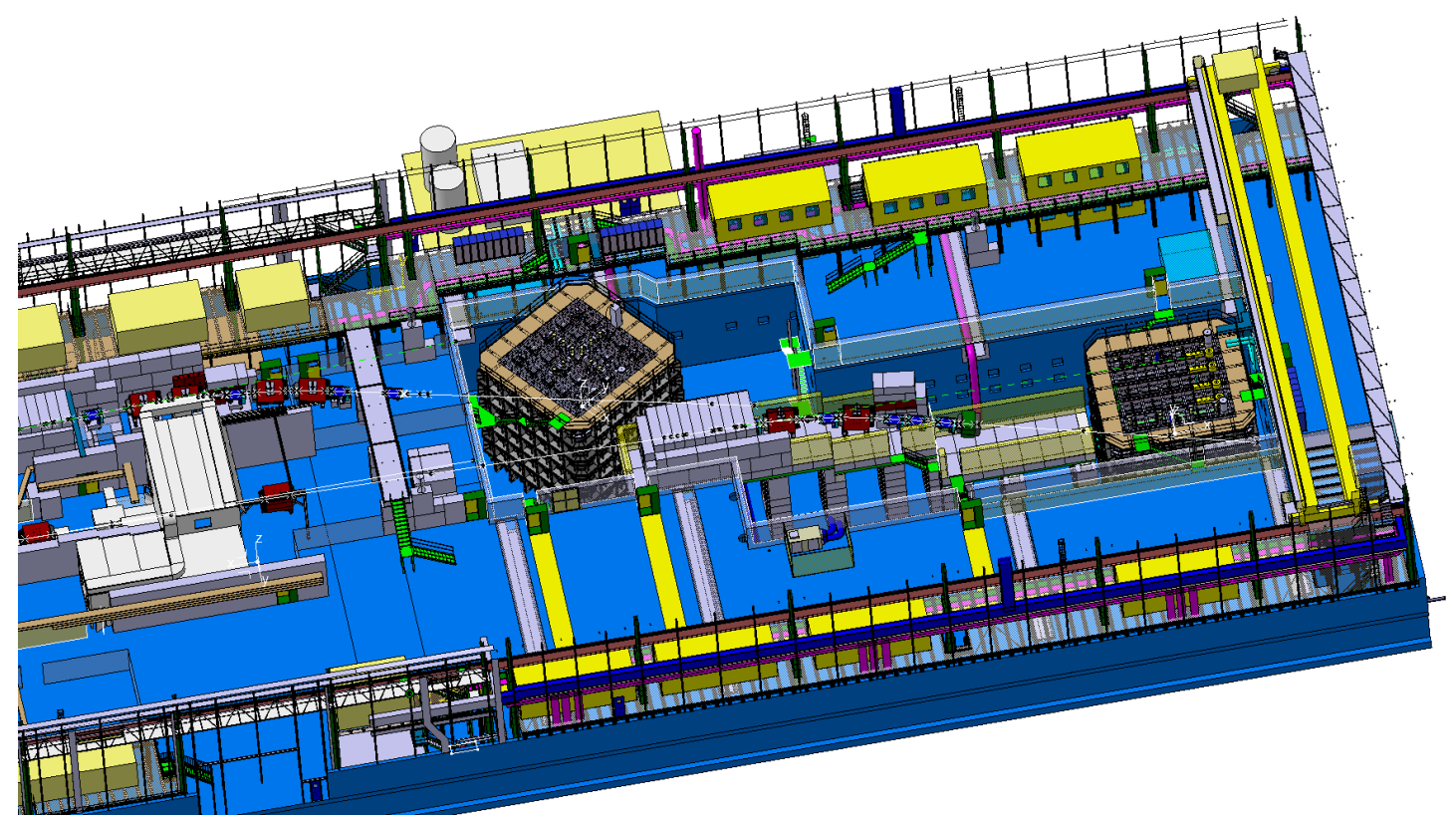

Figure 1: Diagram of the layout of the CERN north area with location of the protoDUNE dual phase detector (center) and the single phase detector (right). The direction of the particle beam is from left to right.

\section{Overview of the protoDUNE-SP experiment}

The protoDUNE program aims to validate various aspects of the DUNE Liquid Argon Time Projection Chamber (LArTPC) technology before proceeding with the construction of the largescale principal DUNE detectors at the Sanford Underground Research Facility [1, 2]. It is designed to make a series of measurements of the interactions of charged particles in the Liquid Argon medium. These measurements will be performed with a dedicated test beam line at the CERN SPS accelerator complex which will allow the transport of beam particles from $\sim 0.5 \mathrm{GeV} / \mathrm{c}$ up to 7 $\mathrm{GeV} / \mathrm{c}$. The run plan also includes a large number of cosmic ray triggers. The program includes two separate large LArTPC prototypes, one based on a "single-phase" (liquid) technology and the other based on a "dual-phase" (liquid/gaseous) TPC readout technology. Both detectors are placed in an extension of the CERN North Area Experimental Hall and scheduled to take data in 2018. The general layout of the experimental area is shown in Fig. 1, with the single-phase detector seen as a cubic structure on the right.

Located in the vicinity of the detectors will be enclosures which will house the elements of the local computing infrastructure (including Data Acquisition, Online Buffer etc). These enclosures are shown schematically as yellow blocks in the upper-right portion of Fig. 1, and they will have a dedicated $20 \mathrm{~Gb} / \mathrm{s}$ network connection over optical fiber to the CERN central storage facilities.

In the following we shall focus on the single-phase prototype which will be referred to as "protoDUNE-SP". It has also received the official CERN designation as the CERN experiment NP04. This device is essentially an ionization chamber in which Liquid Argon serves both as the target and the sensitive medium with which to detect and measure ionization, with subsequent full 3D resonstruction of events of interest. To this end, it is instrumented with a large number 
Table 1: protoDUNE-SP readout parameters

\begin{tabular}{lr}
\hline Detector Parameter & Target \\
\hline TPC channel count & 15,360 \\
Digitization frequency & $2 \mathrm{MHz}$ \\
Nominal electron drift time & $2.2 \mathrm{~ms}$ \\
Nominal electron drift velocity & $1.6 \mathrm{~mm} / \mu \mathrm{s}$ \\
Readout window & $5 \mathrm{~ms}$ \\
SPS spill time & $4.8 \mathrm{~s}$ \\
SPS cycle & $22.5 \mathrm{~s}$ \\
Nominal trigger rate & $25 \mathrm{~Hz}$ \\
Single readout size (per trigger) & $230.4 \mathrm{MB}$ \\
Lossless compression factor (estimated) & 4 \\
Instantaneous data rate (in-spill) & $1440 \mathrm{MB} / \mathrm{s}$ \\
Average data rate & $300 \mathrm{MB} / \mathrm{s}$ \\
Total data recorded (beam + cosmic) & $3 \mathrm{~PB}$ \\
Buffer to store 3 days worth of data & $300 \mathrm{~TB}$ \\
\hline
\end{tabular}

of wire sensors immersed in Liquid Argon and grouped into planar arrays (termed Anode Plane Assemblies, or APAs). Each face of the APA contains two induction planes and one collection plane, in a stereo angle configuration. The protoDUNE-SP LArTPC will contain six APAs of the same design as in the DUNE experiment, and of the same size which is $6 \times 2.4 \mathrm{~m}$. The maximum electron drift distance (i.e. the span between the cathode and the APA) is $3.6 \mathrm{~m}$ and the drift field $500 \mathrm{~V} / \mathrm{cm}$. The fiducial volume of Liquid Argon in the detector is approximately $7.2 \times 7.2 \times 6.0 \mathrm{~m}^{3}$. The cryostat is shaped as a cube with $\sim 11 \mathrm{~m}$ side (external dimensions). The detector features the "cold electronics" design in which the amplifiers and digitizers are placed within the cryostat and operate at cryogenic temperatures. Digital signals are fed to the Warm Interface Boards located outside of the cryostat on its top surface and then transmitted to the Data Acquisition System via a fiber optic line.

An important part of the DUNE design which is also included in protoDUNE-SP is the Photon Detector capable of providing an accurate measurement of the event timing. The components of this detector are integrated into the APAs.

In order to implement the Cosmic Ray Trigger an external system of scintillation counters is installed outside of the cryostat. The signals from the counters are used in the trigger logic and are also read out and also included in the overall data stream created by the DAQ.

The protoDUNE-SP measurement program requires characterization of the beam particles (including tracking) and adequate triggering capabilities. The beamline used in the experiment is instrumented with fiber tracking devices, Time-of-Flight and Cherenkov counters.

\section{Raw Data Parameters in protoDUNE-SP}

The DUNE/protoDUNE single-phase LArTPC design has a fine spatial resolution (with wire 
pitch of $\sim 5 \mathrm{~mm}$ ) and thus a high channel count. The $2 \mathrm{MHz}$ digitization frequency provides similarly fine resolution along the drift axis of the detector. The readout window is set to $5 \mathrm{~ms}$ based on the nominal scale of electron drift time across the active volume $(2.2 \mathrm{~ms})$ to allow for detection of ionization immediately before and after the event of interest. These characteristics are typical of similar modern Liquid Argon TPC detectors. The principal parameters which define the expected data rate and volume in protoDUNE-SP are presented in Table 1.

Combination of the high channel count, projected trigger rate, digitization frequency and the relatively long readout window leads to a substantial data rate and volume. It has been decided that only lossless compression will be applied to the data. Based on the experience of the $\mu$ BooNE experiment [3] the lossless compression factor is conservatively estimated as $\sim 4$. This also implies that before any processing can take place the data will have to be decompressed.

The protoDUNE-SP Data Acquisition System (DAQ) is writing data to a high performance attached storage system capable of reliably handling the data rates as outlined in Table 1. From that point it is transferred to the CERN Central Storage Facility which includes the high-performance distributed disk storage (EOS) and the tape archive (the CASTOR system at CERN) [4]. The data is then transmitted to Fermilab where the bulk of offline processing will take place. All these transfers are managed by an automated service based on Fermi-FTS [5, 6].

\section{Data Quality Monitoring}

The protoDUNE-SP experiment will use an Online Monitoring system which obtains its input data directly and in real time from the DAQ. Its function is provide a very quick assessment of the general detector conditions and to ascertain proper functioning of the readout chain. Online Monitoring characterized by low latency, high bandwidth and limited CPU resources (located in the vicinity of the detector). This puts restrictions on what types of processing can be usefully accomplished in this environment. In order for more complex metrics to be calculated and actionable information to be provided to the experiment operators (such as estimations of the Liquid Argon purity, signal characteristics after noise reduction, correlations of channel noise etc) the experiment will have a separate Data Quality Monitoring (DQM) system which complements the Online Monitoring. This is similar to many High-Energy Physics experiments which implement "express streams" to perform preliminary calculations on the data almost immediately after it leaves the DAQ. DQM is characterized by more relaxed requiremens regarding latency of processing (which can take tens of minutes) and higher scalability, allowing utilization of resources such the CERN Tier- 0 computing facility. It will include tasks such as

- Signal Processing (noise reduction, deconvolution) and basic Visualization in 2D projections

- Liquid Argon purity monitoring using cosmic $\mu$ tracks

- Basic monitoring of the Beam Instrumentation devices

- Matching of beam particle tracks to signals deteected in the TPC

As an example, the workflow described in the first item in the list above can be modeled as a Directed Acyclic Graph (DAG) is illustrated in Fig.2. It contains data decompression and his- 


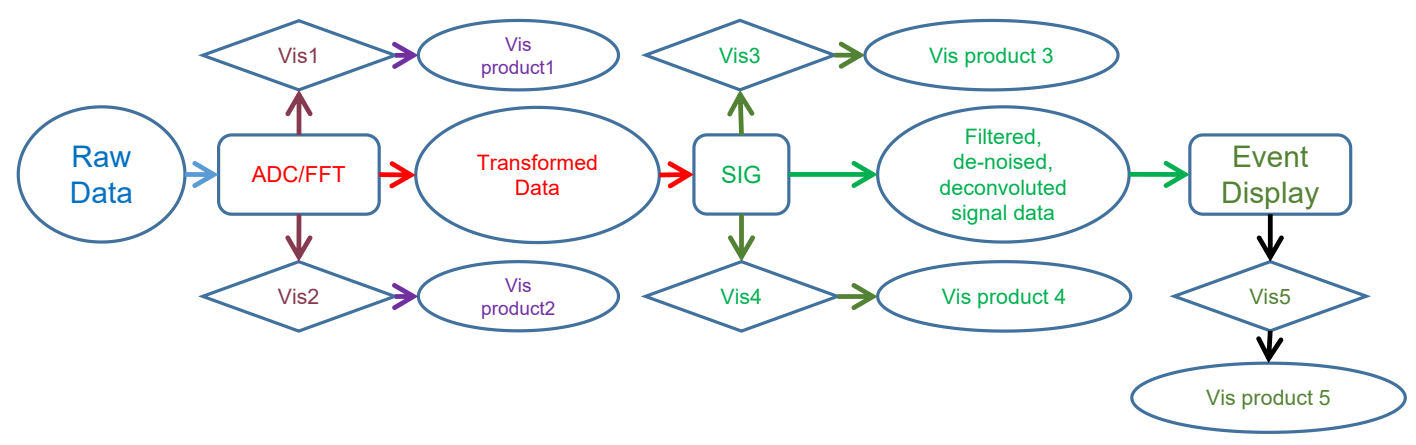

Figure 2: An example of the Data Quality Monitoring workflow

togramming ("ADC"), Fast Fourier Transform stage ("FFT"), application of digital filters and deconvolution algorithms and finally the visualization stage. Each stage in the diagram may produce numerical and graphic data (labeled "vis product" in the diagram) a fraction of which will be persisted in long-term storage for later reference and debugging.

\section{Requirements and Assumptions for the Prompt Processing System}

In order to support the Data Quality Monitoring and to provide automation, efficiency of operation and optimal user experience the "protoDUNE Prompt Processing System" (abbreviated as $p 3 s$ ) has been developed based on the following requirements:

- maximal simplicity of deployment and maintenance

- high degree of automation

- support of computational workflows and their prioritization

- sufficient monitoring capabilities in order to manage and track execution of workflows and ensure quick reponse and corrective actions from the experiment operators

The prompt processing system as described here differs from a typical Workload Management System in a few important aspects, such as

- the data is assumed to be accessible transparently i.e. either reside in a filesystem mounted on the p3s worker nodes, or available via XRootD [7]; this means that data transport aspect in $\mathrm{p} 3 \mathrm{~s}$ is minimal

- to ensure guaranteed execution of high-priority workflows certain jobs and workflows can be automatically removed from the processing pool (i.e. partial loss of results is acceptable depending on the choice of policy) 


\section{The Pilot Framework}

Conceptually p3s is similar to pilot-based Workload Management Systems such as Panda [8], Dirac [9] and others. In this approach, a pilot job (sometimes called an agent) is submitted to a worker node either using a local batch system, or via the Grid, or using any other available submission mechanism. There is no dependence on the type of the local batch system apart from the pilot submission aspect. The pilot can contain useful functionality such as validation of the environment and input data, checking storage availability etc. Subsequently, the pilot connects to the p3s server via HTTP and requests a job to execute.

Jobs descriptions are registered in the $\mathrm{p} 3 \mathrm{~s}$ database as entries containing all necessary information - such as the path to the executable and the configuration file, additional environment variables that need to be set etc. These entries are created independently from pilots by external clients such as the CLI-based client actuated by the user on a remote machine or an agent process which automatically creates job descriptions triggered by arrival of fresh data. All interaction with the server is via HTTP in this case as well.

Upon receiving a request from a pilot job the server picks a job from the database (effectively a queue) based on priorities and responds to the pilot with a message containing the job description. The pilot parses the message (which arrives in JSON format) and initiates the execution. It also periodically checks the state of the job under its management, sending periodic heartbeats to the server. Once the job completes, the pilot repeats the cycle. Its lifetime is usually set to be much larger than typical execution time of jobs run in the system so the next job starts very quickly and avoids the latency that may exist on the underlying batch system.

If the server does not receive heartbeats from the pilot (e.g. due to the pilot exhausting the time allotted by the batch queue) within a period of time over a certain limit such pilot and the job under its management are declared "timed-out" and marked in the database correspondingly. There is a separate process that checks pilot population in $\mathrm{p} 3 \mathrm{~s}$ and automatically submits fresh pilots to keep it at the required level.

\section{Services, Interfaces and Component Reuse}

The client-server architecture is used. The p3s server is implemented as a Django [10] application with standard components such as Apache Web server and PostgreSQL RDBMS as the backend storage (a few other RDBMS can be used as well). All interactions with the server are conducted via HTTP. The server provides monitoring capabilities by allowing the user to browse and navigate tabulated data describing the state of the various objects in the system (e.g. pilots, jobs, data files etc). The design of monitoring pages leverages the well known "django-tables2" package which results in a very small amout of application code.

A suite of CLI clients is provided for managing pilots, jobs and other components. In case interaction with the server requires exhange of messages these are formatted as JSON. Workflows (DAGs) can be described in a standard format (GraphML) which is parsed using the popular NetworkX Python module, again minimizing the application code. 


\section{Deployment}

The protoDUNE Prompt Processing System has been deployed at CERN, with the Web and database services running on the CERN OpenStack virtual machines (CentOS 7). Installation of the server and client application software is straightforward since it is Python code which can be pulled from a repository or installed from a tarball. Python 3.5 is required. Since it has to be installed in the user space on the client machines at CERN (such as running the CLI interface) due to the site policy the Python virtual environment appears to be the optimal solution. The CERN-supported "acrontab" utility which is equivalent to Unix crontab but is optimized for a large Kerberos-authenticated cluster is used to perform periodic maintenance tasks in p3s.

At the time of writing $\mathrm{p} 3 \mathrm{~s}$ is running continuously on the lxbatch facility at CERN. To date, tens of thousands of test jobs have run. Scalability tests are under way utilizing realistic payload jobs developed for protoDUNE.

\section{References}

[1] R. Acciarri et al. Long-Baseline Neutrino Facility (LBNF) and Deep Underground Neutrino Experiment (DUNE) Conceptual Design Report Volume 1: The LBNF and DUNE Projects. e-Print: arXiv:1601.05471

[2] R. Acciarri et al. Long-Baseline Neutrino Facility (LBNF) and Deep Underground Neutrino Experiment (DUNE) Conceptual Design Report, Volume 4: The DUNE Detectors at LBNF. e-Print: arXiv:1601.02984

[3] B. Jones et al. The Status of the MicroBooNE Experiment. J. Phys.: Conf. Series. Vol.408. IOP Publishing, 2013, doi:10.1088/1742-6596/408/1/012028

[4] L. Mascetti et al. Disk storage at CERN. J. Phys.: Conf. Series. Vol.664. IOP Publishing, 2015, doi:10.1088/1742-6596/664/4/042035

[5] R. A. Illingworth A data handling system for modern and future Fermilab experiments. J. Phys.: Conf. Series. Vol.513. IOP Publishing, 2014, doi:10.1088/1742-6596/513/3/032045

[6] A. Norman The Fermilab File Transfer System. e-Print: FNAL CD-DocDB-5412

[7] L. Bauerdick et al. Using Xrootd to Federate Regional Storage. J. Phys.: Conf. Series. Vol.396. IOP Publishing, 2012, doi:10.1088/1742-6596/396/4/042009

[8] T. Maeno et al. Overview of ATLAS PanDA Workload Management. J. Phys.: Conf. Series. Vol.331. IOP Publishing, 2011, doi:10.1088/1742-6596/331/7/072024

[9] A. Casajus et al. DIRAC Pilot Framework and the DIRAC Workload Management System. J. Phys.: Conf. Series. Vol.219. IOP Publishing, 2010, doi:10.1088/1742-6596/219/6/062049

[10] N. George Mastering Django: Core. The Complete Guide to Django 1.8 LTS GNW Independent Publishing, ISBN: 099461683X 